\title{
EXISTENCE AND CONCENTRATION OF SOLUTIONS FOR A CLASS OF ELLIPTIC PROBLEMS WITH DISCONTINUOUS NONLINEARITY IN $\mathrm{R}^{N}$
}

\author{
CLAUDIANOR O. ALVES* and RÚBIA G. NASCIMENTO ${ }^{\dagger}$
}

\begin{abstract}
Using variational methods we establish existence and concentration of positive solutions for a class of elliptic problems in $\mathrm{R}^{N}$, whose nonlinearity is discontinuous.
\end{abstract}

\section{Introduction}

Many recent studies have focused on the nonlinear Schrödinger equation (NLS) $i \epsilon \frac{\partial \Psi}{\partial t}=-\epsilon^{2} \Delta \Psi+(V(x)+E) \Psi-f(\Psi) \quad$ for all $\quad x \in \mathrm{R}^{N}$, where $\epsilon>0$. Knowledge of the solutions for the elliptic equation $\left(P_{\epsilon}\right) \quad-\epsilon^{2} \Delta u+V(x) u=f(u) \quad$ in $\mathrm{R}^{N}$,

which can be rewritten of the form

$$
-\Delta u+V(\epsilon x) u=f(u) \quad \text { in } \quad \mathrm{R}^{N},
$$

has great importance in the study of standing-wave solutions of (NLS). In recent years, the existence and concentration of positive solutions for general semilinear elliptic equations $\left(P_{\epsilon}\right)$ for the case $N \geq 3$ have been extensively studied, see for example, Bartsch, Pankov \& Wang [6], Floer \& Weinstein [17], Oh [21], Rabinowitz [22], Wang [24], Alves \& Souto [5], Alves \& Figueiredo [3], [4], del Pino \& Felmer [15] and their references.

In [22], by a mountain pass argument, Rabinowitz proves the existence of positive solutions of $\left(P_{\epsilon}\right)$, for $\epsilon>0$ small, whenever

$$
V_{\infty}=\liminf _{|x| \rightarrow \infty} V(x)>\inf _{x \in \mathrm{R}^{N}} V(x)=\gamma>0 .
$$

\footnotetext{
* Partially supported by CNPq/Brazil 620150/2008-4 and 303080/2009-4.

${ }^{\dagger}$ Partially supported by CNPq 505407/2008-6.

Received 24 December 2010.
} 
Later Wang [24] showed that these solutions concentrate at global minimum points of $V(x)$ as $\epsilon$ tends to 0 .

In [15], del Pino and Felmer find solutions which concentrate around a local minimum of $V(x)$ by introducing a penalization method. More precisely, they assume that there is an open and bounded set $\Lambda$ compactly contained in $\Omega$ such that

$$
0<\gamma \leq V_{0}=\inf _{x \in \Lambda} V(x)<\min _{x \in \partial \Lambda} V(x) .
$$

In [18], Gazzola \& Radulescu have considered the existence of solution for the following class of problem

$$
\begin{cases}L u+V(x) u=f(x, u) & \text { in } \mathbf{R}^{N}, \\ u>0 & \text { in } \mathbf{R}^{N},\end{cases}
$$

where $L$ is a general elliptic operator of second order and $f$ is a discontinuous function with subcritical growth and $V: \mathrm{R}^{N} \rightarrow \mathrm{R}$ is a coercive continuous function, that is,

$$
V(x) \rightarrow+\infty \quad \text { as } \quad|x| \rightarrow+\infty .
$$

In that paper, by using variational methods, the authors show that the coercivity of $V$ implies that the nonlinearity defines a compact operator. Such a property is crucial to conclude that weak limit of (PS) sequence of the energy functional are solutions of the problem. Still related to elliptic problems with discontinuous nonlinearity, we cite the papers of Alves, Bertone \& Gonçalves [1], Alves, Santos \& Gonçalves [2], Badiale [7], [8], Dinu [16], Radulescu [23] and their references.

Motivated by the papers [18], [22] and [24], we study the existence and concentration of solutions for the following class of problem

$$
(P)_{\epsilon, \beta} \quad \begin{cases}-\Delta u+V(\epsilon x) u=H(u-\beta) u^{p} & \text { in } \mathbf{R}^{N}, \\ u>0 & \text { in } \mathbf{R}^{N},\end{cases}
$$

where $\epsilon, \beta>0$ are positive parameters, $H$ is the Heaviside function given by

$$
H(t)= \begin{cases}1 & \text { if } t>0 \\ 0 & \text { if } t \leq 0\end{cases}
$$

and $p \in\left(1, \frac{N+2}{N-2}\right)$ if $N \geq 3$ or $p \in(1,+\infty)$ if $N=1,2$. Moreover, $V$ : $\mathrm{R}^{N} \rightarrow \mathrm{R}$ is a positive continuous function verifying the assumption $\left(V_{0}\right)$. 
By a solution for $(P)_{\epsilon, \beta}$, we understand as a function $u \in W_{\mathrm{loc}}^{2, \frac{p+1}{p}}\left(\mathrm{R}^{N}\right) \cap$ $H^{1}\left(\mathrm{R}^{N}\right)$ verifying

$$
-\Delta u(x)+V(\epsilon x) u(x) \in[\underline{g}(u(x)), \bar{g}(u(x))] \quad \text { a.e in } \quad \mathrm{R}^{N}
$$

where $g(t)=H(t-\beta) t^{p}, \bar{g}(t)=\lim _{\delta \rightarrow 0^{+}} g(t+\delta)$ and $\underline{g}(t)=\lim _{\delta \rightarrow 0^{+}} g(t-$ $\delta)$.

The interest in the study of nonlinear partial differential equations with discontinuous nonlinearities has increased because many free boundary problems arising in mathematical physics may be stated in this form. Among these problems, we have the obstacle problem, the seepage surface problem, and the Elenbaas equation, see for example [10], [11], [12].

Our main result is the following:

THeOREm 1.1. Assume that $\left(V_{0}\right)$ holds. Then, there are $\epsilon^{*}, \beta^{*}>0$ such that $(P)_{\epsilon, \beta}$ has a positive solution $u_{\epsilon, \beta}$ for $\epsilon \in\left(0, \epsilon^{*}\right)$ and $\beta \in\left(0, \beta^{*}\right)$. Moreover, if $z_{\epsilon, \beta} \in \mathrm{R}^{N}$ denotes a maximum point of $u_{\epsilon, \beta}$, we have that

$$
\lim _{(\epsilon, \beta) \rightarrow(0,0)} V\left(\epsilon z_{\epsilon, \beta}\right)=\gamma .
$$

Theorem 1.1 completes the study made in [18], [22] and [24], in the sense that, in [18] the authors studied the case $\liminf _{|x| \rightarrow \infty} V(x)=\infty$, because in that paper $V$ is coercive. In the present paper, we do not assume this assumption on $V$, this way, $V$ can not be coercive. Once that $V$ is not necessarily coercive, we use a new argument to overcome the lack of compactness involving the nonlinearity, see Lemma 3.3 in Section 3. Related to the papers [22] and [24], our contribution is directly related to the fact that we are considering a discontinuous nonlinearity, while that in those papers, the nonlinearity considered was continuous. The authors believe that this is the first work related to the study of concentration of the solutions for a class of elliptic problems with discontinuous nonlinearity. A first difficulty involving this class of problem, is the fact that we cannot use the classical variational methods; here it is necessary to use some results for a locally Lipschitz functional. When the nonlinearity is continuous and satisfies some assumptions, the mountain pass level is equal to the minimum of the energy functional on Nehari manifolds, which is a key point in a lot of papers. However, this property is not true for discontinuous nonlinearity. Hence, the arguments used in the above reference cannot be repeated directly, and a careful analysis is necessary to get similar results to those found in [22] and [24]. 


\section{Basic results from convex analysis}

In this section, for the reader's convenience, we recall some definitions and basic results on the critical point theory of locally Lipschitz continuous functionals as developed by Chang [10], Clarke [13], [14] and Grossinho \& Tersin [19].

Let $X$ be a real Banach space. A functional $I: X \rightarrow \mathrm{R}$ is locally Lipschitz continuous, $I \in \operatorname{Lip}_{\text {loc }}(X, \mathrm{R})$ for short, if given $u \in X$ there is an open neighborhood $V:=V_{u} \subset X$ and some constant $K=K_{V}>0$ such that

$$
\left|I\left(v_{2}\right)-I\left(v_{1}\right)\right| \leq K\left\|v_{2}-v_{1}\right\|, \quad v_{i} \in V, \quad i=1,2 .
$$

The directional derivative of $I$ at $u$ in the direction of $v \in X$ is defined by

$$
I^{0}(u ; v)=\limsup _{h \rightarrow 0, \lambda \downarrow 0} \frac{I(u+h+\lambda v)-I(u+h)}{\lambda} .
$$

Hence $I^{0}(u ;$.$) is continuous, convex and its subdifferential at z \in X$ is given by

$$
\partial I^{0}(u ; z)=\left\{\mu \in X^{*} ; I^{0}(u ; v) \geq I^{0}(u ; z)+\langle\mu, v-z\rangle, v \in X\right\},
$$

where $\langle.,$.$\rangle is the duality pairing between X^{*}$ and $X$. The generalized gradient of $I$ at $u$ is the set

$$
\partial I(u)=\left\{\mu \in X^{*} ;\langle\mu, v\rangle \leq I^{0}(u ; v), v \in X\right\} .
$$

Since $I^{0}(u ; 0)=0, \partial I(u)$ is the subdifferential of $I^{0}(u ; 0)$.

A few definitions and properties will be recalled below.

$$
\begin{gathered}
\partial I(u) \subset X^{*} \text { is convex, non-empty and weak*-compact, } \\
\lambda(u)=\min \left\{\|\mu\|_{X^{*}} ; \mu \in \partial I(u)\right\},
\end{gathered}
$$

and

$$
\partial I(u)=\left\{I^{\prime}(u)\right\}, \quad \text { if } \quad I \in C^{1}(X, \mathrm{R}) .
$$

A critical point of $I$ is an element $u_{0} \in X$ such that $0 \in \partial I\left(u_{0}\right)$ and a critical value of $I$ is a real number $c$ such that $I\left(u_{0}\right)=c$ for some critical point $u_{0} \in X$.

Theorem 2.1 ([19], [23]). Let $I \in \operatorname{Lip}_{\text {loc }}(X, \mathrm{R})$ with $I(0)=0$ and satisfying:

(i) There are $r>0$ and $\rho>0$, such that $I(u) \geq \rho$, for $\|u\|=r, u \in X$;

(ii) There is $e \in X \backslash B_{r}(0)$ with $I(e)<0$. 
If $c=\inf _{\gamma \in \Gamma} \max _{t \in[0,1]} I(\gamma(t))$ and

$$
\Gamma=\{\gamma \in C([0,1], X), \gamma(0)=0 \text { and } \gamma(1)=e\},
$$

then $c \geq \rho$ and there is a sequence $\left\{u_{n}\right\} \subset X$ verifying

$$
I\left(u_{n}\right) \rightarrow c \quad \text { and } \quad \lambda\left(u_{n}\right) \rightarrow 0 .
$$

Proposition 2.1 ([10], [13]). Let $\left\{u_{n}\right\} \subset X$ and $\left\{\rho_{n}\right\} \subset X^{*}$ with $\rho_{n} \in$ $\partial I\left(u_{n}\right)$. If $u_{n} \rightarrow u$ in $X$ and $\rho_{n} \stackrel{*}{\rightarrow} \rho$ in $X^{*}$, then $\rho_{0} \in \partial I(u)$.

Proposition 2.2 ([10], [13]). Let $R>0, \Psi(u)=\int_{\mathrm{R}^{N}} G(u) d x$ and $\Psi_{R}(v)=\int_{B_{R}(0)} G(v) d x$, where $G(t)=\int_{0}^{t} g(s) d s$. Then, $\Psi \in \operatorname{Lip}_{\text {loc }}\left(L^{p+1}\left(\mathrm{R}^{N}\right)\right.$, $\mathrm{R}), \Psi_{R} \in \operatorname{Lip}_{\text {loc }}\left(L^{p+1}\left(B_{R}(0)\right), \mathrm{R}\right), \partial \Psi(u) \in L^{\frac{p+1}{p}}\left(\mathrm{R}^{N}\right)$ and $\partial \Psi_{R}(v) \in$ $L^{\frac{p+1}{p}}\left(B_{R}(0)\right)$. Moreover, if $\rho \in \partial \Psi(u)$ and $\zeta \in \partial \Psi_{R}(v)$, then

$$
\rho(x) \in[\underline{g}(u(x)), \bar{g}(u(x))] \quad \text { a.e in } \mathrm{R}^{N}
$$

and

$$
\zeta(x) \in[\underline{g}(v(x)), \bar{g}(v(x))] \quad \text { a.e in } \quad B_{R}(0) .
$$

\section{Existence of solution for $(P)_{1, \beta}$}

In this section, we will suppose that $V_{\infty}<+\infty$, because the case $V_{\infty}=+\infty$ has been considered in [18]. Here, we consider the space

$$
E=\left\{u \in H^{1}\left(\mathrm{R}^{N}\right) ; \int_{\mathrm{R}^{N}} V(x) u^{2}<\infty\right\}
$$

endowed with the norm

$$
\|u\|^{2}=\int_{\mathrm{R}^{N}}\left(|\nabla u|^{2}+V(x) u^{2}\right),
$$

which is associated with inner product

$$
\langle u, v\rangle=\int_{\mathrm{R}^{N}}(\nabla u \nabla u+V(x) u v) .
$$

The energy functional associated with $(P)_{1, \beta}$ is given by

$$
I_{\beta}(u)=\frac{1}{2} \int_{\mathrm{R}^{N}}\left(|\nabla u|^{2}+V(x) u^{2}\right)-\int_{\mathrm{R}^{N}} G(u),
$$

where $G(t)=\int_{0}^{t} g(s) d s$. 
LeMma 3.1. The functional $I_{\beta}$ satisfies the mountain pass geometry.

Proof. We begin observing that $I_{\beta}(0)=0$ and

$$
I_{\beta}(u) \geq \frac{1}{2}\|u\|^{2}-\frac{1}{p+1} \int_{\Omega}|u|^{p+1} .
$$

Hence, by Sobolev embedding

$$
I_{\beta}(u) \geq \frac{1}{2}\|u\|^{2}-C\|u\|^{p+1} .
$$

Thereby, there exist $r, \rho>0$ verifying

$$
I_{\beta}(u) \geq \rho>0 \quad \text { for } \quad\|u\|=r, u \in E .
$$

Now, let $\psi \in C_{0}^{\infty}\left(\mathrm{R}^{N}\right)$ with $\psi>0$ and $K=\operatorname{supt} \psi \subset \mathrm{R}^{N}$. Then, for $t>0$ $I_{\beta}(t \psi) \leq \frac{t^{2}}{2}\|\psi\|^{2}-\frac{t^{p+1}}{p+1} \int_{K \cap[t \psi>\beta]} \psi^{p+1}+\int_{K \cap[t \psi>\beta]} \frac{\beta^{p+1}}{p+1}+C \operatorname{med}(K)$.

Once that $p>1$,

$$
I_{\beta}(t \psi) \rightarrow-\infty \quad \text { as } \quad t \rightarrow+\infty
$$

Consequently, for $t_{0}>0$ large enough, we derive that $e=t_{0} \psi$ satisfies

$$
I_{\beta}(e)<0 \quad \text { with } \quad e \in E \backslash B_{r}(0) .
$$

Combining Lemma 3.1 with Theorem 2.1 , there is a sequence $\left\{u_{n}\right\} \subset E$ satisfying

$$
I_{\beta}\left(u_{n}\right) \rightarrow c_{\beta} \quad \text { and } \quad \lambda_{\beta}\left(u_{n}\right) \rightarrow 0,
$$

where $c_{\beta}$ is the mountain pass level of the functional $I_{\beta}$.

In what follows, we will show that $\left\{u_{n}\right\}$ given in (3.3) is bounded in $E$. To this end, we will use the following notation for the functional $I_{\beta}$ :

$$
I_{\beta}(u)=Q(u)-\Psi(u)
$$

where $Q(u)=\frac{1}{2} \int_{\mathrm{R}^{N}}\left(|\nabla u|^{2}+V(x) u^{2}\right)$ and $\Psi(u)=\int_{\mathrm{R}^{N}} G(u)$.

LEMMA 3.2. The sequence $\left\{u_{n}\right\}$ is bounded in $E$.

Proof. From (3.3),

$$
I_{\beta}\left(u_{n}\right) \rightarrow c_{\beta} \quad \text { and } \quad \lambda_{\beta}\left(u_{n}\right) \rightarrow 0
$$


From now on, we consider $\left\{\omega_{n}\right\} \subset\left(H^{1}\left(\mathrm{R}^{N}\right)\right)^{*}$ such that $\lambda_{\beta}\left(u_{n}\right)=\left\|\omega_{n}\right\|_{*}$ and

$$
\omega_{n}=Q^{\prime}\left(u_{n}\right)-\rho_{n}
$$

where $\left\{\rho_{n}\right\} \subset \partial \Psi\left(u_{n}\right)$. Then,

$$
\left\langle\omega_{n}+\rho_{n}, u_{n}\right\rangle=\left\langle Q^{\prime}\left(u_{n}\right), u_{n}\right\rangle=\left\|u_{n}\right\|^{2} .
$$

Once that $0 \leq(p+1) G(t) \leq t \underline{g}(t) \forall t \in \mathrm{R}$, it follows that

$$
\Psi\left(u_{n}\right)=\int_{\mathrm{R}^{N}} G\left(u_{n}\right) \leq \frac{1}{p+1} \int_{\mathrm{R}^{N}} u_{n} \underline{g}\left(u_{n}\right) .
$$

From Proposition 2.2,

$$
\underline{g}\left(u_{n}(x)\right) \leq \rho_{n}(x) \leq \bar{g}\left(u_{n}(x)\right) \quad \text { a.e in } \quad \mathbf{R}^{N}
$$

leading to

$$
u_{n}(x) \underline{g}\left(u_{n}(x)\right) \leq \rho_{n}(x) u_{n}(x) \quad \text { a.e in } \quad \mathrm{R}^{N},
$$

which implies that

$$
\int_{\mathrm{R}^{N}} u_{n} \underline{g}\left(u_{n}\right) \leq \int_{\mathrm{R}^{N}} \rho_{n} u_{n}=\left\langle\rho_{n}, u_{n}\right\rangle .
$$

Therefore,

$$
\Psi\left(u_{n}\right) \leq \frac{1}{p+1} \int_{\mathrm{R}^{N}} u_{n} \underline{g}\left(u_{n}\right) \leq \frac{1}{p+1}\left\langle\rho_{n}, u_{n}\right\rangle .
$$

Combining (3.4) and (3.5),

$$
I_{\beta}\left(u_{n}\right)-\frac{1}{p+1}\left\langle\omega_{n}+\rho_{n}, u_{n}\right\rangle \geq\left(\frac{1}{2}-\frac{1}{p+1}\right)\left\|u_{n}\right\|^{2}-\frac{1}{p+1}\left\langle\rho_{n}, u_{n}\right\rangle,
$$

or equivalently,

$$
I_{\beta}\left(u_{n}\right)-\frac{1}{p+1}\left\langle\omega_{n}, u_{n}\right\rangle \geq\left(\frac{1}{2}-\frac{1}{p+1}\right)\left\|u_{n}\right\|^{2} .
$$

Using the fact that $\left\{I_{\beta}\left(u_{n}\right)\right\}$ is bounded, there is $M>0$ such that

$$
M-\frac{1}{p+1}\left\langle\omega_{n}, u_{n}\right\rangle \geq\left(\frac{1}{2}-\frac{1}{p+1}\right)\left\|u_{n}\right\|^{2} .
$$


Recalling that $\left\langle\omega_{n}, u_{n}\right\rangle=o_{n}(1)$, we have

$$
M+o_{n}(1)\left\|u_{n}\right\| \geq\left(\frac{1}{2}-\frac{1}{p+1}\right)\left\|u_{n}\right\|^{2},
$$

showing that $\left\{u_{n}\right\}$ is bounded in $E$.

The next lemma is a key point in our argument, because the functional $\Psi$ is not compact. In what follows, for each $R>0$, we set $\Psi_{R}: L^{p+1}\left(B_{R}(0)\right) \rightarrow \mathrm{R}$ the following functional

$$
\Psi_{R}(u)=\int_{B_{R}(0)} G(u) .
$$

Moreover, for each $\psi \in L^{p+1}\left(B_{R}(0)\right)$, we set the function $\tilde{\psi} \in L^{p+1}\left(\mathrm{R}^{N}\right)$ given by

$$
\tilde{\psi}(x)= \begin{cases}\psi(x), & x \in B_{R}(0) \\ 0, & x \in B_{R}^{c}(0) .\end{cases}
$$

Lemma 3.3. Let $\left\{u_{n}\right\} \subset E$ with $u_{n} \rightarrow u$ in $E$ and $\left\{\rho_{n}\right\} \subset \partial \Psi\left(u_{n}\right)$ with $\rho_{n} \rightarrow \rho_{0}$ in $L^{\frac{p+1}{p}}\left(\mathrm{R}^{N}\right)$. Then,

$$
\rho_{0}(x) \in[\underline{g}(u(x)), \bar{g}(u(x))] \quad \text { a.e in } \mathrm{R}^{N} .
$$

Proof. Hereafter, for each $R>0$, we denote by $u_{n, R}, \rho_{n, R}, u_{R}$ and $\rho_{0, R}$ the restriction of the functions $u_{n}, \rho_{n}, u$ and $\rho_{0}$ to $B_{R}(0)$. For each $\psi \in$ $L^{p+1}\left(B_{R}(0)\right)$, a simple computation implies that

$$
\int_{B_{R}(0)} \rho_{n, R} \psi=\int_{\mathrm{R}^{N}} \rho_{n} \tilde{\psi}
$$

and

$$
\Psi_{R}^{0}\left(u_{n, R}, \psi\right)=\Psi^{0}\left(u_{n}, \tilde{\psi}\right)
$$

Once that

$$
\int_{\mathrm{R}^{N}} \rho_{n} \tilde{\psi} \leq \Psi^{0}\left(u_{n}, \tilde{\psi}\right)
$$

we derive

$$
\int_{B_{R}(0)} \rho_{n, R} \psi \leq \Psi_{R}^{0}\left(u_{n, R}, \psi\right), \quad \forall \psi \in L^{p+1}\left(B_{R}(0)\right),
$$

which yields

$$
\rho_{n, R} \in \partial \Psi_{R}\left(u_{n, R}\right) .
$$


Since, $u_{n, R} \rightarrow u_{R}$ in $L^{p+1}\left(B_{R}(0)\right)$ and $\rho_{n, R} \rightarrow \rho_{0, R}$ in $L^{\frac{p+1}{p}}\left(B_{R}(0)\right)$, from Proposition 2.1

$$
\rho_{0, R} \in \partial \Psi_{R}\left(u_{R}\right)
$$

and so, by Proposition 2.2

$$
\rho_{0, R}(x) \in\left[\underline{g}\left(u_{R}(x)\right), \bar{g}\left(u_{R}(x)\right)\right] \quad \text { a.e in } \quad B_{R}(0),
$$

or equivalently,

$$
\rho_{0}(x) \in[\underline{g}(u(x)), \bar{g}(u(x))] \quad \text { a.e in } \quad B_{R}(0) .
$$

Now, using the fact that $R>0$ is arbitrary, we get

$$
\rho_{0}(x) \in[\underline{g}(u(x)), \bar{g}(u(x))] \quad \text { a.e in } \mathrm{R}^{N} .
$$

THeOREM 3.1. Assume that $c_{\beta}<c_{\infty}$, where $c_{\infty}$ is the mountain pass level associated with the functional

$$
I_{\infty}(u)=\frac{1}{2} \int_{\mathrm{R}^{N}}\left(|\nabla u|^{2}+V_{\infty} u^{2}\right)-\frac{1}{p+1} \int_{\mathrm{R}^{N}}|u|^{p+1}, \quad \forall u \in H^{1}\left(\mathrm{R}^{N}\right) .
$$

Then, $(P)_{1, \beta}$ has a nontrivial solution.

Proof. From Lemma 3.1 and Theorem 2.1, there is a sequence $\left\{u_{n}\right\} \subset E$ satisfying

$$
I_{\beta}\left(u_{n}\right) \rightarrow c_{\beta} \quad \text { and } \quad \lambda_{\beta}\left(u_{n}\right) \rightarrow 0 .
$$

By using standard arguments, we can assume, without loss of generality, that $\left\{u_{n}\right\}$ is bounded in $E$ and $u_{n}(x) \geq 0$ for all $x \in \mathrm{R}^{N}$. This way, there is $u \in H^{1}\left(\mathrm{R}^{N}\right)$ such that, for some subsequence,

$$
u_{n} \rightarrow u \quad \text { in } E
$$

and

$$
u_{n} \rightarrow u \quad \text { in } \quad L_{\mathrm{loc}}^{p}\left(\mathrm{R}^{N}\right) .
$$

Claim 3.1. The weak limit $u$ is nontrivial, that is, $u \neq 0$.

In fact, if $u \equiv 0$, the limit $u_{n} \rightarrow 0$ in $E$ does not hold, because $c_{\beta}>0$. From Lions' lemma [20], there are $\left\{y_{n}\right\} \subset \mathrm{R}^{N}$ and $r, \alpha>0$ verifying

$$
\liminf _{n \rightarrow \infty} \int_{B_{r}\left(y_{n}\right)}\left|u_{n}\right|^{2} \geq \alpha>0 .
$$


Since we are supposing $u=0$, the Sobolev embedding combined with the above inequality yields $\left\{y_{n}\right\}$ is unbounded. Now, we set

$$
v_{n}(x)=u_{n}\left(x+y_{n}\right) .
$$

Using the boundedness of $\left\{u_{n}\right\}$ in $E$, it follows that $\left\{v_{n}\right\}$ is bounded in $H^{1}\left(\mathrm{R}^{N}\right)$. Hence, there is $v \in H^{1}\left(\mathrm{R}^{N}\right) \backslash\{0\}$ and a subsequence of $\left\{v_{n}\right\}$, still denote by itself, such that

$$
v_{n} \rightarrow v \quad \text { in } \quad E
$$

and

$$
v_{n} \rightarrow v \quad \text { in } \quad L_{\mathrm{loc}}^{s}\left(\mathrm{R}^{N}\right),
$$

$1 \leq s<2^{*}$ if $N \geq 3$ and $1 \leq s$ if $N=1,2$.

Let $\varphi \in C_{0}^{\infty}\left(\mathrm{R}^{N}\right)$ verifying $\varphi(x)=1$ for $x \in B_{1}(0), \varphi(x)=0$ for $x \in$ $B_{2}^{c}(0), 0 \leq \varphi(x) \leq 1$ and $\varphi_{R}(x)=\varphi\left(\frac{x}{R}\right)$ for $R>0$. Then,

$$
\left\langle\omega_{n},\left(\varphi_{R} v_{n}\right)\left(\cdot-y_{n}\right)\right\rangle=o_{n}(1),
$$

because the sequence $\left\{\left(\varphi_{R} v_{n}\right)\left(\cdot-y_{n}\right)\right\}$ is bounded in $E$. Thereby,

$$
\begin{aligned}
\int_{\mathrm{R}^{N}} \nabla u_{n} \nabla\left(\left(\varphi_{R} u_{n}\right)\left(x-y_{n}\right)\right)+V u_{n} & \left(\varphi_{R} v_{n}\right)\left(x-y_{n}\right) \\
& =\int_{\mathrm{R}^{N}} \rho_{n}\left(\left(\varphi_{R} v_{n}\right)\left(x-y_{n}\right)\right)+o_{n}(1),
\end{aligned}
$$

and so,

$$
\int_{B_{2 R}}\left|\nabla v_{n}\right|^{2} \varphi_{R}(x)+\int_{B_{2 R}} V\left(x+y_{n}\right) v_{n}^{2} \varphi_{R}+\int_{B_{2 R}} v_{n} \nabla v_{n} \nabla \varphi_{R} \leq \int_{B_{2 R}} v_{n}^{p+1} \varphi_{R} .
$$

Combining Fatou's lemma with the Sobolev embedding, we get

$$
\int_{B_{2 R}}|\nabla v|^{2} \varphi_{R}+\int_{B_{2 R}} V_{\infty} v^{2} \varphi_{R}+\int_{B_{2 R}} v \nabla v \nabla \varphi_{R} \leq \int_{B_{2 R}} v^{p+1} \varphi_{R} .
$$

Now, taking the limit of $R \rightarrow+\infty$,

$$
\int_{\mathrm{R}^{N}}|\nabla v|^{2}+V_{\infty} v^{2} \leq \int_{\mathrm{R}^{N}} v^{p+1} .
$$

Once that $v \neq 0$, there is $t>0$, such that $t v \in \mathcal{N}_{\infty}$, where $\mathcal{N}_{\infty}$ is the Nehari manifold associated with $I_{\infty}$ given by

$$
\mathscr{N}_{\infty}=\left\{u \in H^{1}\left(\mathrm{R}^{N}\right) \backslash\{0\} ; I_{\infty}^{\prime}(u) u=0\right\} .
$$


Then,

$$
\int_{\mathrm{R}^{N}}|\nabla v|^{2}+V_{\infty} v^{2}=t^{p-1} \int_{\mathrm{R}^{N}} v^{p+1}
$$

From (3.10) and (3.11), we conclude that $t \leq 1$.

By a result found in Willem [25],

$$
c_{\infty} \leq \inf _{u \in \mathcal{N}_{\infty}} I_{\infty}(u)
$$

from where it follows that $c_{\infty} \leq I_{\infty}(t v)$. Consequently,

$$
\begin{aligned}
c_{\infty} \leq I_{\infty}(t v) & =I_{\infty}(t v)-\frac{1}{p+1} I_{\infty}^{\prime}(t v) t v \\
& =\left(\frac{1}{2}-\frac{1}{p+1}\right) t^{2} \int_{\mathrm{R}^{N}}|\nabla v|^{2}+V_{\infty} v^{2}
\end{aligned}
$$

and since $t \leq 1$,

$$
c_{\infty} \leq\left(\frac{1}{2}-\frac{1}{p+1}\right) \int_{\mathrm{R}^{N}}|\nabla v|^{2}+V_{\infty} v^{2}
$$

Combining Fatou's lemma with the inequality $\underline{g}(s) s \geq(p+1) G(s)$ for all $s \geq 0$, we obtain

$$
\begin{aligned}
c_{\infty} \leq \lim \inf \left[\left(\frac{1}{2}-\frac{1}{p+1}\right) \int_{\mathrm{R}^{N}}\left|\nabla u_{n}\right|^{2}\right. & +V(x) u_{n}^{2} \\
& \left.+\int_{\mathrm{R}^{N}}\left(\frac{g\left(u_{n}\right) u_{n}}{p+1}-G\left(u_{n}\right)\right)\right] .
\end{aligned}
$$

By Proposition 2.2,

$$
\begin{aligned}
c_{\infty} \leq \liminf _{n \rightarrow+\infty}\left[\left(\frac{1}{2}-\frac{1}{p+1}\right) \int_{\mathrm{R}^{N}}\left|\nabla u_{n}\right|^{2}\right. & +V(x) u_{n}^{2} \\
& \left.+\frac{1}{p+1} \int_{\mathrm{R}^{N}} \rho_{n} u_{n}-\int_{\mathrm{R}^{N}} G\left(u_{n}\right)\right]
\end{aligned}
$$

that is,

$$
c_{\infty} \leq \liminf _{n \rightarrow+\infty}\left[I_{\beta}\left(u_{n}\right)-\frac{1}{p+1}\left\langle\omega_{n}, u_{n}\right\rangle\right]=\liminf _{n \rightarrow+\infty}\left[I_{\beta}\left(u_{n}\right)+o_{n}(1)\right]=c_{\beta},
$$


obtaining a contradiction. Therefore, $u \geq 0$ and $u \neq 0$. Next, we will show that $u$ is a solution for $(P)_{1, \beta}$. To this end, we need to prove that $u \in W_{\text {loc }}^{2, \frac{p+1}{p}}\left(\mathrm{R}^{N}\right) \cap$ $H^{1}\left(\mathrm{R}^{N}\right)$ and

$$
-\Delta u(x)+V(x) u(x) \in[\underline{g}(u(x)), \bar{g}(u(x))] \quad \text { a.e in } \quad \mathrm{R}^{N} .
$$

Since $\left\{u_{n}\right\} \subset E$ is a $(P S)_{c_{\beta}}$ sequence, there are $\left\{\omega_{n}\right\} \subset \partial I_{\beta}\left(u_{n}\right)$ and $\left\{\rho_{n}\right\} \subset$ $\partial \Psi\left(u_{n}\right)$ verifying

$$
\left\|\omega_{n}\right\|_{*} \rightarrow 0
$$

and

$$
\left\langle\omega_{n}, \varphi\right\rangle=\int_{\mathrm{R}^{N}} \nabla u_{n} \nabla \varphi+V(x) u_{n} \varphi-\int_{\mathrm{R}^{N}} \rho_{n} \varphi, \quad \varphi \in E,
$$

with

$$
\rho_{n}(x) \in\left[\underline{g}\left(u_{n}(x)\right), \bar{g}\left(u_{n}(x)\right)\right] \quad \text { a.e in } \quad \mathrm{R}^{N} .
$$

The boundedness of $\left\{u_{n}\right\}$ combined with (3.14) implies that $\left\{\rho_{n}\right\}$ is bounded in $L^{\frac{p+1}{p}}\left(\mathrm{R}^{N}\right)$. Thus, there is $\rho_{0} \in L^{\frac{p+1}{p}}\left(\mathrm{R}^{N}\right)$ and a subsequence of $\left\{\rho_{n}\right\}$, such that

$$
\rho_{n} \rightarrow \rho_{0} \quad \text { in } \quad L^{\frac{p+1}{p}}\left(\mathbf{R}^{N}\right) .
$$

From (3.7) and (3.15)

$$
\int_{\mathrm{R}^{N}} \nabla u \nabla \varphi+V(x) u \varphi=\int_{\mathrm{R}^{N}} \rho_{0} \varphi, \quad \forall \varphi \in E .
$$

Moreover, by Lemma 3.3,

$$
\rho_{0} \in[\underline{g}(u(x)), \bar{g}(u(x))] \quad \text { a.e in } \quad \mathrm{R}^{N},
$$

showing that $u$ is a nonnegative weak solution of the problem

$$
-\Delta u+V(x) u=\rho_{0} \quad \text { in } \quad \mathrm{R}^{N} .
$$

Using regularity theory, it follows that $u \in W_{\mathrm{loc}}^{2, \frac{p+1}{p}}\left(\mathrm{R}^{N}\right)$, and so

$$
-\Delta u(x)+V(x) u(x)=\rho_{0}(x) \quad \text { a.e in } \quad \mathrm{R}^{N} .
$$

Therefore, from (3.16) and (3.17) it follows that $u$ is a solution for $(P)_{1, \beta}$. 
REMARK 3.1. Using the fact that $V(x) \geq \gamma$ for all $x \in \mathrm{R}^{N}$, it is immediate to check, by using the Stampachia theorem, that $\left\{x \in \mathrm{R}^{N} ; u(x)=\beta\right\}$ has null measure for $\beta$ small enough, this way, the solution $u$ verifies

$$
-\Delta u+V(x) u=H(u-\beta) u^{p} \quad \text { a.e in } \quad \mathrm{R}^{N} .
$$

This information is very important in many applications.

\section{Existence and concentration of solutions for $(P)_{\epsilon, \beta}$}

In this section, we consider the space

$$
E_{\epsilon}=\left\{u \in H^{1}\left(\mathrm{R}^{N}\right) ; \int_{\mathrm{R}^{N}} V(\epsilon x) u^{2}<\infty\right\}
$$

endowed with the norm

$$
\|u\|^{2}=\int_{\mathrm{R}^{N}}\left(|\nabla u|^{2}+V(\epsilon x) u^{2}\right),
$$

which is associated with inner product

$$
\langle u, v\rangle=\int_{\mathrm{R}^{N}}(\nabla u \nabla u+V(\epsilon x) u v) .
$$

The energy functional associated with $(P)_{\epsilon, \beta}$ is given by

$$
I_{\epsilon, \beta}(u)=\frac{1}{2} \int_{\mathrm{R}^{N}}\left(|\nabla u|^{2}+V(\epsilon x) u^{2}\right)-\int_{\mathrm{R}^{N}} G(u),
$$

and its mountain pass level is denoted by $c_{\epsilon, \beta}$.

Proof of Theorem 1.1. We will divide the proof in two steps.

Step 1: Existence. In what follows, $\omega \in H^{1}\left(\mathrm{R}^{N}\right)$ denotes a positive ground state solution of the problem

$$
\begin{cases}-\Delta \omega+\gamma \omega=\omega^{p} & \text { in } \mathbf{R}^{N}, \\ \omega>0 & \text { in } \mathbf{R}^{N},\end{cases}
$$

that is, if $I_{0}: H^{1}\left(\mathrm{R}^{N}\right) \rightarrow \mathrm{R}$ is the functional energy associated with (4.19) given by

$$
I_{0}(u)=\frac{1}{2} \int_{\mathrm{R}^{N}}|\nabla u|^{2}+\gamma|u|^{2}-\frac{1}{p+1} \int_{\mathrm{R}^{N}}|u|^{p+1},
$$

we have that $I_{0}(\omega)=c_{0}$ and $I_{0}^{\prime}(\omega)=0$, where $c_{0}$ is the mountain pass level. 
Hereafter, we set $\varphi \in C_{0}^{\infty}\left(\mathrm{R}^{N}\right)$ verifying

$0 \leq \varphi(x) \leq 1, \varphi(x)=1 \quad \forall x \in B_{1}(0) \quad$ and $\quad \varphi(x)=0 \quad \forall x \in B_{2}^{c}(0)$.

Furthermore, for each $R>1$, we denote by $\varphi_{R}$ and $\omega_{R}$ the functions

$$
\varphi_{R}(x)=\varphi\left(\frac{x}{R}\right) \quad \text { and } \quad \omega_{R}(x)=\varphi_{R}(x) \omega(x) .
$$

A direct computation shows that

$$
\omega_{R} \rightarrow \omega \text { in } \quad H^{1}\left(\mathrm{R}^{N}\right) \quad \text { as } \quad R \rightarrow+\infty .
$$

Hence, $\omega_{R} \neq 0$ for $R$ large enough. From this, there is $t_{R}>0$, such that

$$
I_{0}\left(t_{R} w_{R}\right)=\max _{t \geq 0} I_{0}\left(t \omega_{R}\right)
$$

and so,

$$
\int_{B_{2 R}}\left|\nabla \omega_{R}\right|^{2}+\gamma\left|\omega_{R}\right|^{2}=t_{R}^{p-1} \int_{B_{2 R}} \omega_{R}^{p+1}
$$

and

$$
\lim _{R \rightarrow \infty} t_{R}=1
$$

These facts imply that

$$
\hat{\omega}_{R}=\omega_{R} t_{R} \rightarrow \omega \text { in } \quad H^{1}\left(\mathrm{R}^{N}\right) \quad \text { as } \quad R \rightarrow \infty .
$$

Once that $c_{0}<c_{\infty}$, see [22], we can choose $\delta, R>0$ such that

$$
c_{0}+\delta<c_{\infty} \quad \text { and } \quad I_{0}\left(\hat{\omega}_{R}\right)<c_{0}+\frac{\delta}{2},
$$

and $t_{*}>0$ verifying $I_{\epsilon, \beta}\left(t_{*} \hat{\omega}_{R}\right)<0$ uniformly in $\epsilon, \beta>0$ small enough.

Now, we consider $\hat{\gamma}(t)=t\left(t_{*} \hat{\omega}_{R}\right)$ for $t \in[0,1]$, which belongs to $\hat{\gamma} \in \Gamma$. From the definition of $c_{\epsilon, \beta}$

$$
c_{\epsilon, \beta} \leq \max _{t \in[0,1]} I_{\epsilon, \beta}(\hat{\gamma}(t))=\max _{t \geq 0} I_{\epsilon, \beta}\left(t \hat{\omega}_{R}\right)=I_{\epsilon, \beta}\left(\hat{t} \hat{\omega}_{R}\right)
$$

for some $\hat{t}=\hat{t}(\epsilon, \beta, R)>0$.

A straightforward computation implies that for each $R>0$ given, there are positive constants $C, K$ such that $C \leq \hat{t} \leq K$, for $\epsilon, \beta>0$ small enough.

Now, since $\gamma \leq V(x)$ for all $x \in \mathrm{R}^{N}$, it is easy to check that

$$
c_{0} \leq c_{\epsilon, \beta} \leq \max _{t \geq 0} I_{\epsilon, \beta}\left(t \hat{\omega}_{R}\right) .
$$


Without loss of generality, we assume that $V(0)=\gamma$. Thus, for each $\eta>0$, there is $\epsilon_{0}>0$ such that,

$$
0<V(\epsilon x)-\gamma<\eta \quad \text { for } \quad \epsilon \in\left(0, \epsilon_{0}\right) \text { and } x \in \operatorname{supt} \omega_{R}=B_{2 R}(0),
$$

from where it follows that

$$
\int_{\mathrm{R}^{N}} V(\epsilon x) \hat{\omega}_{R}^{2}<\int_{\mathrm{R}^{N}}(\gamma+\eta) \hat{\omega}_{R}^{2}
$$

Using these informations,

$c_{\epsilon, \beta} \leq I_{0}\left(\hat{t} \hat{\omega}_{R}\right)+\frac{\hat{t}^{2}}{2} \eta \int_{B_{2 R}} \hat{\omega}_{R}^{2}+\frac{\hat{t}^{p+1}}{p+1} \int_{B_{2 R} \cap\left[\hat{t} \hat{\omega}_{R} \leq \beta\right]} \hat{\omega}_{R}^{p+1}+\int_{B_{2 R} \cap\left[\hat{t} \hat{\omega}_{R}>\beta\right]} \frac{\beta^{p+1}}{p+1}$,

that is,

$$
c_{\epsilon, \beta} \leq c_{0}+\frac{\delta}{2}+\eta C_{1}+C_{2} \beta^{p+1}
$$

where $C_{1}, C_{2}$ do not depend of $\epsilon, \beta>0$. Hence, for $\eta, \beta>0$ small enough

$$
c_{\epsilon, \beta} \leq c_{0}+\frac{\delta}{2}+\frac{\delta}{4}+\frac{\delta}{4} \leq c_{0}+\delta<c_{\infty}
$$

From Theorem 3.1, the problem $(P)_{\epsilon, \beta}$ has a nontrivial solution for $\epsilon, \beta>0$ small enough.

Step 2: Concentration of the solutions. Hereafter, we denote by $u_{\epsilon, \beta}$ the solution given by Step 1. Thereby, there exists $\rho_{\epsilon, \beta} \in L^{\frac{p+1}{p}}\left(\mathrm{R}^{N}\right)$ such that

$$
-\Delta u_{\epsilon, \beta}(x)+V(x) u_{\epsilon, \beta}(x)=\rho_{\epsilon, \beta}(x) \quad \text { a.e in } \quad \mathrm{R}^{N}
$$

with $\rho_{\epsilon, \beta} \in\left[g\left(u_{\epsilon, \beta}(x)\right), \bar{g}\left(u_{\epsilon, \beta}(x)\right)\right]$ a.e in $\mathbf{R}^{N}$.

Next, we fix $\epsilon_{n} \rightarrow 0, \beta_{n} \rightarrow 0, u_{n}=u_{\epsilon_{n}, \beta_{n}}$ and $\rho_{n}=\rho_{\epsilon_{n}, \beta_{n}}$. Our goal is to study the behavior of the maximum points related to $\left\{u_{n}\right\}$, more precisely, if $z_{n} \in \mathrm{R}^{N}$ denotes a maximum point of $u_{n}$, we will prove that

$$
\lim _{n \rightarrow \infty} V\left(\epsilon_{n} z_{n}\right)=\gamma
$$

The arguments used in the proof of Step 1, more precisely in (4.22) and (4.23), lead to

$$
\lim _{n \rightarrow \infty} c_{\epsilon_{n}, \beta_{n}}=c_{0}>0 .
$$

Claim 4.1. There are $\left\{y_{n}\right\} \subset \mathbf{R}^{N}$ and $r, \eta>0$ such that

$$
\liminf _{n \rightarrow \infty} \int_{B_{r}\left(y_{n}\right)}\left|u_{n}\right|^{2} \geq \eta>0 .
$$


In fact, if the claim does not hold, then by a result due to Lions

$$
\lim _{n \rightarrow \infty} \int_{\mathrm{R}^{N}}\left|u_{n}\right|^{p+1}=0 .
$$

This limit combined with the fact that $u_{n}$ is a solution of $(P)_{\epsilon_{n}, \beta_{n}}$ implies that

$$
\lim _{n \rightarrow \infty} c_{\epsilon_{n}, \beta_{n}}=\lim _{n \rightarrow \infty} I_{\epsilon_{n}, \beta_{n}}\left(u_{n}\right)=0,
$$

which is a contradiction with (4.25).

CLAIM 4.2. The sequence $v_{n}=u_{n}\left(\cdot-y_{n}\right)$ is strongly convergent in $H^{1}\left(\mathrm{R}^{N}\right)$. Moreover,

$$
\lim _{|x| \rightarrow \infty} v_{n}(x)=0
$$

uniformly in $n \in \mathrm{N}$, that is, for each $\eta>0$, there is $R>0$ such that

$$
\left|v_{n}(x)\right|<\eta \quad \forall x \in \mathrm{R}^{N} \backslash B_{R}(0) \text { and } n \in \mathrm{N} .
$$

In fact, repeating the same arguments used in the proof of Theorem 3.1, more precisely Claim 3.1, we can assume without loss of generality that $\left\{\epsilon_{n} y_{n}\right\}$ is a convergent sequence in $\mathrm{R}^{N}$ with $\epsilon_{n} y_{n} \rightarrow y^{*} \in V^{-1}(\gamma)$. Furthermore, we also derive that if $v$ is the weak limit of $\left\{v_{n}\right\}$, then

$$
v_{n} \rightarrow v \quad \text { in } \quad H^{1}\left(\mathrm{R}^{N}\right) .
$$

Now, following the arguments found in Alves \& Figueiredo [3], the Moser interaction implies that

$$
\lim _{|x| \rightarrow \infty} v_{n}(x)=0
$$

uniformly in $n \in \mathrm{N}$. The basic idea to prove the last limit is to consider the following functions

$$
\begin{aligned}
u_{L, n}(x) & = \begin{cases}u_{n}(x), & u_{n}(x) \leq L \\
L, & u_{n}(x) \geq L\end{cases} \\
z_{L, n} & =\eta_{n}^{p} u_{L, n}^{p(\xi-1)} u_{n}
\end{aligned}
$$

with $\xi>1$ to be determined later. The limit in (4.26) follows taking $z_{L, n}\left(\cdot-y_{n}\right)$ as a test function in (4.24) combined with the inequality $\rho_{n}(x) \leq u_{n}^{p}(x)$ a.e in $\mathrm{R}^{N}$. 
On the other hand, it follows from (4.25) that $\lim _{\inf } \operatorname{lo}_{n \rightarrow \infty}\left\|v_{n}\right\|_{\infty, \mathrm{R}^{N}}>0$, then there are $\delta^{*}>0$ and $n_{0} \in \mathrm{N}$ such that

$$
\left\|v_{n}\right\|_{\infty, \mathrm{R}^{N}} \geq \delta^{*}, \quad \forall n \geq n_{0} .
$$

Choosing $\eta=\frac{\delta^{*}}{2}$, there is $R>0$ such that

$$
v_{n}(x)<\frac{\delta^{*}}{2} \quad \forall x \in \mathrm{R}^{N} \backslash B_{R}(0) \quad \text { and } \quad n \in \mathrm{N}
$$

and so, if $x_{n}$ denotes a maximum point of $v_{n}$, we get

$$
v_{n}\left(x_{n}\right) \geq \delta^{*} \quad \text { and } \quad x_{n} \in B_{R}(0) .
$$

Now, if $z_{n}$ denote the maximum point of $u_{n}$, we have that $z_{n}=x_{n}+y_{n}$, this way, $\epsilon_{n} z_{n}=\epsilon_{n} x_{n}+\epsilon_{n} y_{n} \rightarrow y^{*}$. Since $V$ is a continuous function,

$$
\lim _{n \rightarrow \infty} V\left(\epsilon_{n} z_{n}\right)=V\left(y^{*}\right)=\gamma
$$

\section{REFERENCES}

1. Alves, C. O., Bertone, A. M., and Gonçalves, J. V., A variational approach to discontinuous problems with critical Sobolev exponents, J. Math. Anal. App. 265 (2002), 103-127.

2. Alves, C. O., Santos, J. A., and Gonçalves, J. V., On multiple solutions for multivalued elliptic equations under Navier boundary conditions, J. Convex Anal. 18 (2011), 627-644.

3. Alves, C. O., and Figueiredo, G. M., Existence and multiplicity of positive solutions to a p-Laplacian equation in $\mathbf{R}^{N}$, Differential Integral Equations 19 (2006), 143-162.

4. Alves, C. O., and Figueiredo, G. M., Multiplicity of positive solutions for a quasilinear problem in $\mathrm{R}^{N}$ via penalization method, Adv. Nonlinear Stud. 5 (2005), 551-572.

5. Alves, C. O., and Souto, M. A. S., On existence and concentration behavior of ground state solutions for a class of problems with critical growth, Commun. Pure Appl. Anal. 1 (2002), 417-431.

6. Bartsch, T., Pankov, A., and Wang, Z.-Q., Nonlinear Schrödinger equations with steep potential well, Commun. Contemp. Math. 3 (2001), 549-569.

7. Badiale, M., Critical exponent and discontinuous nonlinearities, Differential Integral Equations 6 (1993), 1173-1185.

8. Badiale, M., Some remarks on elliptic problems with discontinuous nonlinearities, Rend. Sem. Mat. Univ. Politec. Torino 51 (1993), 331-342.

9. Brézis, H., and Lieb, E. H., A relation between pointwise convergence of functions and convergence of functionals, Proc. Amer. Math. Soc. 8 (1983), 486-490.

10. Chang, K. C., Variational methods for nondifferentiable functionals and their applications to partial differential equations, J. Math. Anal. 80 (1981), 102-129.

11. Chang, K. C., On the multiple solutions of the elliptic differential equations with discontinuous nonlinear terms, Sci. Sinica 21 (1978), 139-158.

12. Chang, K. C., The obstacle problem and partial differential equations with discontinuous nonlinearities, Comm. Pure Appl. Math. 33 (1980), 117-146.

13. Clarke, F. H., Optimization and Nonsmooth Analysis, Wiley, New York 1983. 
14. Clarke, F. H., Generalized gradients and applications, Trans. Amer. Math. Soc. 205 (1975), 247-262.

15. del Pino, M. and Felmer, P. L., Local mountain pass for semilinear elliptic problems in unbounded domains, Calc. Var. Partial Differential Equations 4 (1996), 121-137.

16. Dinu, T. L., Standing wave solutions of Schrödinger systems with discontinuous nonlinearity in anisotropic media, Int. J. Math. Math. Sci. 2006, article ID 73619, 13 pages.

17. Floer, A., and Weinstein, A., Nonspreading wave packets for the cubic Schrödinger equation with bounded potential, J. Funct. Anal. 69 (1986), 397-408.

18. Gazzola, F., and Radulescu, V., A nonsmooth critical point theory approach to some nonlinear elliptic equations in $\mathrm{R}^{N}$, Differential Integral Equations 13 (2000), 47-60.

19. Grossinho, M. R., and Tersian, S. A., An Introduction to Minimax Theorems and their Applications to Differential Equations, Nonlinear Optimization Appl. 52, Kluwer, Dordrecht 2001.

20. Lions, P. L., The concentration-compactness principle in the calculus of variations. The locally compact case II, Ann. Inst. H. Poincaré Anal. Non Linéaire 1 (1984), 223-283.

21. Oh, Y.-G., Existence of semi-classical bound states of nonlinear Schrödinger equations with potentials on the class $(V)_{a}$, Comm. Partial Differential Equations 13 (1988), 1499-1519.

22. Rabinowitz, P. H., On a class of nonlinear Schrödinger equations, Z. Angew. Math. Phys. 43 (1992), 270-291.

23. Radulescu, V., Mountain pass theorems for nondifferentiable functions and applications, Proc. Japan. Acad. (A) 69 (1993), 193-198.

24. Wang, X., On concentration of positive bound states of nonlinear Schrödinger equations, Comm. Math. Phys. 153 (1993), 229-244.

25. Willem, M., Minimax Theorems, Progr. Nonlinear Diff. Eq. Appl. 24, Birkhäuser, Boston 1996.

UNIVERSIDADE FEDERAL DE CAMPINA GRANDE

UNIDADE ACADÊMICA DE MATEMÁTICA E ESTATÍSTICA

CEP: 58429-900, CAMPINA GRANDE-PB

BRAZIL

E-mail: coalves@dme.ufcg.edu.br
FACULDADE DE MATEMÁTICA

UNIVERSIDADE FEDERAL DO PARÁ

CEP: 66075-110, BELÉM-PA

BRAZIL

E-mail: rubia@ufpa.br 